\title{
Feasibility of screening all neonates for hearing loss
}

\author{
D M B HALL AND J GARNER
}

\author{
Department of Child Health, St George's Hospital, London
}

SUMmaRY We investigated the feasibility and cost of screening all neonates for hearing loss using the auditory response cradle (ARC). At least three full time staff are needed to screen $95 \%$ of the 3000 infants delivered each year including those in intensive care. Estimated costs per case detected are between $£ 3000$ and $£ 6000$ but true costs may be higher.

The screening of all neonates for congenital sensorineural hearing loss is an attractive proposition, because this is the only time before school entry when one has a 'captive population'. Furthermore, early diagnosis and intervention may improve the prognosis for speech and language development. ${ }^{1}$

The auditory response cradle (ARC) (Interface (Research) Ltd) is a screening device which analyses the neonate's behavioural responses to a standardised sound stimulus. ${ }^{2}$ The purpose of the present study was to determine the resources needed to screen all neonates born in hospital.

\section{Methods}

The project was carried out, by one full time screener (JG), over a 12 month period in a teaching hospital with 3000 births each year. An ARC Mk 6 was used and established procedures were followed. ${ }^{34}$ Babies were not tested on the first day of life.

Every mother was given a leaflet about the test. If the test could not be performed before discharge nursing staff offered the mother an appointment. The screener recorded all births, details of tests performed, and in the case of babies not tested, the reason.

Subsequently, a half time screener was appointed for the regional neonatal intensive care unit (NICU). Data on babies in the NICU were collected over a five month period.

The following assumptions were made in calculating costs: $(a)$ that the cost of the ARC was $£ 4400$ depreciated over five years; $(b)$ that the screener's salary was $£ 6000$ a year; and $(c)$ that there were no charges for test room, printing, stationery, postage or telephone. We also assumed that a consultant audiological service was available for definitive diagnosis and management; that the incidence of congenital sensorineural hearing loss is $1 / 1000(1 / 100$ in babies on a NICU); that the ARC detects all cases; and that none would otherwise be detected in the neonatal period.

\section{Results}

During the main study period, 2821 full term babies were available for testing and 1293 were screened. In 109 cases the first test was abandoned, usually because the child was restless. Twenty seven babies required a second test having failed a complete first test. The total number of tests was 1429. Eight babies were referred for audiological assessment and two had a unilateral hearing loss. No case of bilateral sensorineural hearing loss was found by screening. One mother whose first child had a familial hearing loss was referred directly to the audiologist who confirmed a bilateral sensorineural hearing loss.

The reasons why the remaining 1528 babies were not tested are summarised in the table. An important constraint was the fluctuation in the number of babies who required testing each day (figure). The mean number of tests performed each day was $6 \cdot 8$ (range 4-11). The average screening capacity of one tester was exceeded by the number of births on 99 out of 184 days. Appointments for ARC testing after discharge were offered to 122 mothers but only half attended.

In the study on babies in the NICU, 134 babies were admitted of whom 15 died; this left 119 available for study. Seventy one were tested. Four-

Table Reason why babies were not tested $(n=1528)$

\begin{tabular}{ll}
\hline Reason & No of babies \\
\hline Insufficient time & 426 \\
Discharged at weekend & 244 \\
Admitted and discharged at weekend & 243 \\
Screener took annual leave & 169 \\
Early discharge & 131 \\
Cradle faulty & 114 \\
Statutory holiday & 69 \\
Mother. failed to keep appointment & 61 \\
Mother refused & 40 \\
Screener on sick leave & 21 \\
Miscellaneous & 10 \\
\hline
\end{tabular}




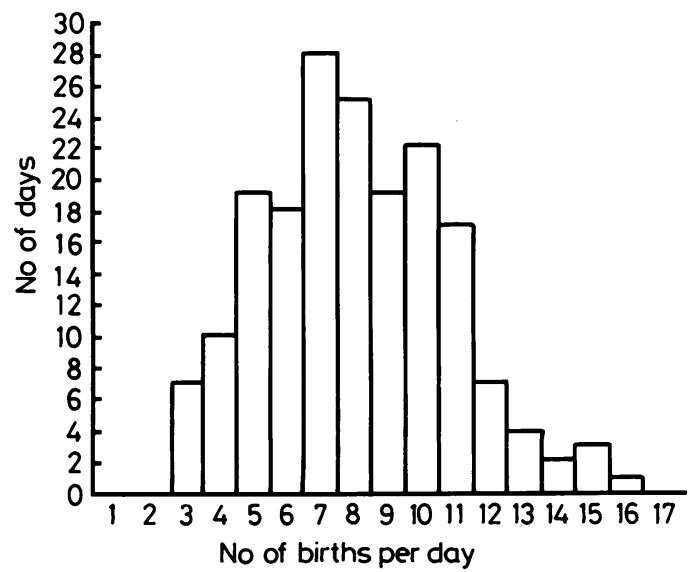

Figure Distribution of the number of births each day over a six month period.

teen needed a repeat test, three were referred, and one had bilateral sensorineural hearing loss. The 48 not tested were either transferred to referring hospitals or still required intensive care at the conclusion of the study.

\section{Discussion}

The two most important constraints on neonatal screening were the fluctuations in the number of births each day and our hospital's early discharge policy. Forty per cent of babies are now discharged before 48 hours of age and an increasing number on the first day.

Although the average time per test was only 30 minutes, testing was often delayed because the baby was crying, restless, or being fed, changed, or examined. It is more realistic to measure the efficiency of an ARC programme in terms of babies tested per week; one full time screener can test between 30 and 40 babies a week.

We thought it would be easier to organise ARC tests in the NICU because babies are often in the unit for long periods, but as soon as the respiratory state was stable the baby, though still too small for an ARC test, was likely to be transferred to the referring hospital.

We estimated the staff needed to screen $95 \%$ of babies. Two screeners would be essential on any day when more than eight babies required testing, and one would have to work over bank holidays and weekends. Because of the need for a flexible rota, holiday cover, and time off in lieu for weekend working, three people would be required working at least two and a half full time equivalents. An additional half time equivalent would be needed to include babies in the NICU. Two screeners can share one ARC, but two machines make for greater efficiency.

The cost of detecting one case of bilateral sensorineural hearing loss in full term infants exceeds $£ 5000$. The overall cost per case falls to between $£ 3000$ and $£ 4000$ if babies in the NICU are included and falls further if babies with unilateral sensorineural hearing loss are counted as cases, but unilateral hearing loss does not cause language delay or require treatment so detection in the neonate is of questionable importance.

Generous assumptions were made in calculating these figures. In reality the reliability, sensitivity, and specificity of the ARC are not established ${ }^{5}$; false negatives do occur; and some cases are found by other means, as in our study. The true cost per case is therefore higher.

Neonatal hearing screening of all babies born in hospital is expensive and presents serious logistic difficulties, which would apply equally to other screening techniques such as brain stem evoked response audiometry or cochlear emission. ${ }^{6}$ The ideal method would be one which the health visitor could use at home, but no such method is currently available. Whether the value of detecting sensorineural hearing loss in the neonate justifies the costs of a screening programme, and whether such a programme can ever replace the seven month screening test are matters for further debate.

We thank Drs Graham and Raglan, Mrs M Cutbill, Miss M Doran, and the staff of the neonatal, obstetric, and audiology departments for their interest and support, and the Special Trustees of St George's Hospital for financial help.

\section{References}

1 Elliot LL, Armbrusher VB. Some possible effects of the delay of early treatment of deafness. J Speech Hear Res 1967;110: 209-24.

2 Bennett MJ. The auditory response cradle: a device for the objective assessment of auditory state in the neonate. Symposia of the Zoological Society of London 1975;37:291-305.

${ }^{3}$ Bhattacharya J, Bennet MJ, Tucker SM. Long term follow up of newborns tested with the auditory response cradle. Arch Dis Child 1984;59:504-11.

${ }^{4}$ McCormick B, Curnock DA, Spavins F. Auditory screening of special care neonates using the auditory response cradle. Arch Dis Child 1984;59:1168-72.

5 Davis A. Detecting hearing impairment in neonates - the statistical decision criterion for the auditory response cradle. $\mathrm{Br}$ J Audiol 1984;18:163-8.

${ }^{6}$ Bray P, Kemp D. An advanced cochlear echo technique suitable for infant screening. Br J Audiol 1987;21:191-204.

Correspondence to Dr DMB Hall, Department of Child Health, St George's Hospital, Tooting, SW17 0RE.

Accepted 3 February 1988 